\title{
Effects of maternal age on the expression of mesenchymal stem cell markers in the components of human umbilical cord
}

\author{
Ghadeer I. Alrefaei ${ }^{1}$, Nasra N. Ayuob ${ }^{2,3}$, Soad S. Ali ${ }^{2}$, Saleh Al-Karim ${ }^{1,4}$ \\ ${ }^{1}$ Biology Department, Faculty of Sciences, King Abdulaziz University, Jeddah, Saudi Arabia \\ ${ }^{2}$ Anatomy Department, Faculty of Medicine, King Abdulaziz University, Jeddah, Saudi Arabia \\ ${ }^{3}$ Histology and Cytology Department, Mansoura University, Mansoura, Egypt \\ ${ }^{4}$ Embryonic Stem Cell Unit, King Fahad Medical Research Center, King Abdulaziz University, \\ Jeddah, Saudi Arabia
}

\begin{abstract}
Introduction. Although the human umbilical cord (UC) has been previously considered a medical waste, its use as a main source of fetal stem cells for regenerative medicine applications has increased over the past few years. The aim of the study was to assess the impact of the maternal age on the expression of mesenchymal stem cells (MSC) markers CD105 and CD29 in the different areas of human UC.

Material and methods. In this comparative cross sectional study, one hundred term UCs from five maternal age groups (20-45 years) were collected after delivery from healthy mothers and were processed to assess both immuno- and gene expression of CD105 and CD29 surface antigen markers using immunohistochemical and RT-PCR techniques. Results. The immunoexpression of CD105 and CD29 in the amniotic membrane (AM) and Wharton's jelly (WJ), the umbilical artery (UA) and the umbilical vein (UV) showed significant negative correlation with the maternal age $(\mathrm{p}<0.001)$. Reduced amount of cells as well as the studied MSC markers and their gene expression levels were documented in older age mothers. CD105-positive MSCs were more abundant in the UA, whereas CD29-positive MSCs were more abundant in the AM and WJ.

Conclusion. The decreased expression of CD105 and CD29 MSCs markers with age suggests that selective isolation of MSCs from Wharton's jelly, umbilical artery or umbilical vein of younger mothers should be recommended. (Folia Histochemica et Cytobiologica 2015, Vol. 53, No. 3, 259-271)
\end{abstract}

Key words: mothers' age; umbilical cord; MSC; CD29; CD105; RT-PCR; IHC

\section{Introduction}

Studies carried out during the last decades strongly supported the concept that the umbilical cord (UC) is an important stem cell source for biomedical research and clinical applications [1]. The use of stem cells from fetal, cord blood and extra-embryonic tissues

Correspondence address: Dr. N. Ayuob

Department of Anatomy

Faculty of Medicine, King Abdulaziz University

Jeddah, Saudi Arabia

tel.: 00966530112205, fax: 009666400855

e-mail: nasraayuob@gmail.com,nayuob@kau.edu.sa for regenerative medicine applications has increased over the past decade. Moreover, because the fetal stem cells can be expanded to very large numbers compared with the adult stem cells, they are ideal candidates for seeding scaffolds and matrices used for tissue engineering applications such as the repair of hollow and solid organs [2]. The UC vessels (one vein and two arteries) and the surrounding mucous connective tissue named Wharton's jelly (WJ) are derived from the embryonic and/or extra-embryonic mesoderm. It was speculated that the UC matrix material is derived from a primitive mesenchyme, which is in a transition state towards the adult bone marrow mesenchyme [3]. 
Human mesenchymal stem cells (MSCs) have been identified and isolated from sub-endothelial layer of umbilical vein (UV) [4], Wharton's jelly of the umbilical cord $[5,6]$ and umbilical cord perivascular layer [7]. These cells are commonly characterized by the expression, in $\geq 95 \%$ of the MSC population, of CD105, CD73, CD90 CD29, CD44, CD51 and CD71 membrane antigens, and the lack of expression of CD45, CD34, CD14, CD11b, CD79 $\alpha$ or CD19 and HLA class II molecules $[8,9]$. It remains unclear whether these cells, isolated from UV, WJ and UA represent distinct cell populations [10]. It was reported that the MSCs isolated from WJ share similar properties with other cord blood MSCs as well as adult bone marrow MSCs in relation to the expression of markers, differentiation potential and cytokines' production $[5,11]$.

The umbilical cord mesenchymal stem cells (UCMSCs) have several advantages making them attractive to the regenerative medicine such as availability, fewer ethical consideration and favorable therapeutic potential [13]. The MSCs of the WJ were specifically investigated in the in vitro and pre-clinical studies for their clinical translational potential $[5,12,14]$.

Many levels of regulation in response to local, systemic, and environmental factors regulate stem cell behavior [15]. Evidence from several tissues also suggests that the stem cell functional output is altered during aging [16]. The effect of aging on the regenerative ability of living organisms and its impact on biological activities has been the focus of many previous studies $[17,18]$ but few, if any, authors have investigated the effect of age on either fetal stem cells in general or specifically on umbilical cord stem cells. Therefore, this study aimed to assess the impact of maternal age on the expression of some MSCs surface antigen markers such as CD105 and CD29 in Wharton's jelly, the umbilical artery and the umbilical vein of the term human umbilical cord. We hypothesized that MSCs marker expression is reduced in umbilical cords of older mothers compared to the younger ones and this reduction is attributed to reduced gene expression as well as cell number.

\section{Material and methods}

Patients' selection. The present study was approved by the Biomedical Research Ethics Committee of the Faculty of Medicine of King Abdulaziz University in Jeddah, Saudi Arabia, and informed consents signed by all participants were obtained. As this was the first study aimed at assessing the impact of maternal age on the stem cell expression in umbilical cord and because of the limited resources, a total of 100 full-term Saudi pregnant mothers were included in this study. The physiological deliveries or those through
Cesarean sections done in the Department of Obstetrics and Gynecology at King Abdulaziz University Hospital, Jeddah, between June, 2012 and May, 2013 were included in this study. The eligibility criteria for the cases were the following: full term ( $\geq$ C37 weeks) pregnant female aged twenty years or higher. The gestational age was confirmed using the date of the last menstrual period and through an ultrasound examination performed 11-13 weeks after gestation [19] obtained from patient's medical records. To ensure that only normal pregnancies were included in the study, the exclusion criteria were the following: pregnancies complicated by gestational diabetes, preeclampsia, or any other chronic diseases. A total of 20 full-term freshly delivered UCs were collected in each of the maternal age groups: A (20-25 years), B (26-30 years), C (31-35 years), D (36-40 years) and E (41-45 years). The predictor, in this study, is the maternal age while the immuno- and gene-expression of stem cell markers are the outcome variables. The potential confounders were eliminated through the exclusion criteria.

Tissue sampling. Each UC collected at the time of delivery was weighted, washed with phosphate-buffered saline (PBS) ( $\mathrm{pH}=7.4, \mathrm{BDH}$, Poole, UK), injected with formalin through the umbilical vein, preserved in formalin and then transferred to the laboratory. The UC was separated from the placenta at its insertion; then the proximal part of $5 \mathrm{~cm}$ length was taken and further sectioned into $0.5 \mathrm{~cm}$ longitudinal sections. The latter were processed to obtain paraffin blocks which were cut serially ( $4 \mu \mathrm{m}$ thick), mounted on glass slides and stained with hematoxylin and eosin [20].

Immunohistochemistry. Human endoglin/CD105 antibody (Leica Bio Systems; Newcastle, UK, diluted to $15 \mu \mathrm{g} / \mathrm{mL}$ ), human integrin $\beta 1 / \mathrm{CD} 29$ antibody (Leica Bio Systems, diluted to $15 \mu \mathrm{g} / \mathrm{mL}$ ), human CD34 antibody (Leica Bio Systems, diluted to $25 \mu \mathrm{g} / \mathrm{mL}$ ) and HRP-conjugated primary antibody dilution buffer ( $R$ \& D System, Minneapolis, MN, USA) were used. The corresponding biotinylated conjugated secondary antibody from a Dako staining system was also used (Dako, Glostrup, Denmark).

The immunohistochemical staining were performed on paraffin-embedded tissue sections according to the procedure previously described [21-23]. Briefly, the sections were deparaffinized using xylene and ethanol. Antigen retrieval was achieved by boiling the tissue slides with $0.01 \mathrm{M}$ citric buffer in a microwave for $5 \mathrm{~min}$. Hydrogen peroxide $(0.5 \%)$ was applied at room temperature (RT) for $10 \mathrm{~min}$ to quench the endogenous peroxidase activity. After blocking for 10 min with $10 \%$ horse serum-Tris buffer for 20 minutes at $\mathrm{RT}$, the sections were incubated with the primary antibody at the dilution noted above at RT for $2 \mathrm{~h}$ and, thereafter, with the secondary antibody for $2 \mathrm{~h}$. Slides stained only with a non-specific $\mathrm{IgG}$ were used as negative controls. The nuclei were counterstained with Mayer's hematoxylin. The 
brown DAB staining of the nuclei, cytoplasm or both was considered a positive staining. An Olympus microscope BX-51 (Olympus, Tokyo, Japan) with a digital camera connected to a computer was used to photograph the images. In order to quantify the immunoexpression of the markers, the area percentage (AP) of the brown label was assessed as an indicator of the cell number while the mean intensity (MI) of the reaction was assessed as an indicator of the expression level. Both parameters were assessed by an investigator who was not aware with the age group examined, in 20 non-overlapping fields in each sample with a $40 \times$ objective lens and a $10 \times$ ocular lens using the Pro Plus image analysis software version 6.0 (Cybernetics, Warrendale, PA, USA) as was described by Decaestecker et al. [24].

Gene expression analysis. Quantitative Real Time Polymerase Chain Reaction (QPCR) was performed in Molecular Biology Lab, Zagazig University, Zagazig, Egypt, to detect the expression of human CD105 and CD29 genes in all examined groups relative to the housekeeping gene, $\beta$-actin. Specimens from the different studied areas of the UC were rapidly dissected immediately after delivery and stored at $-80^{\circ} \mathrm{C}$ until assays were performed. Total RNA was isolated using an RNeasy Mini kit including DNase I digestion following the manufacturer's protocol (\#74104, Qiagen, Hilden, Germany). The RNA was then reverse transcribed using Quantiscript reverse transcriptase (Quanti Tect Reverse Transcription Kit (\#205310, Qiagen) and quantitative RT-PCR analysis was performed with a (Rotor-Gene Q 2plex, Qiagen) and SYBR Green master mix (Roche Diagnostics, Carlsbad, CA, USA). The following primers were used for analysis: CD29, 5'-AATGGAGTGAATGGGACAGG-3' and 5'-TCTGTGAAGCCCAGAGGTTT-3' and CD105, 5'-TCTACCTCCACCATGCCAAGT-3' and 5'-TTTTTCCGCTGTGGTGATGA-3'; $\beta$-actin, 5'-TGTCACCAACTGGGACGATA-3', and 5'-GGGGTGTTGAAGGTCTCAAA-3'. The mRNA concentrations of all detected genes were normalized and represented as a ratio against the expression of $A C T B$. The software (Rotor Gene Q, version 2.0.2, Build 4, Qiagen) was used for data analysis.

Statistical analysis. The data was analyzed using the Statistical Package for the Social Sciences (SPSS, version 16, Chicago, IL, USA) software. For the analysis of quantitative non-parametric data, Kruskal-Wallis analysis of variance (ANOVA) followed by a post-hoc test (based on the Dunnett's $C$ procedure) was used to analyze each pair of groups to avoid a multiple-comparison effect. For the quantitative parametric data, the different groups were compared using ANOVA (F-test), followed by a Bonferroni post-hoc test. Results were expressed as mean \pm standard deviation (SD) as well as the lower and upper bounds of $95 \%$ confidence interval for mean. The correlation of the maternal age with the immunoexpression of MSCs markers in different parts
Table 1. Maternal age and fetal mass in the different age groups

\begin{tabular}{|l|c|c|}
\hline Age groups & $\begin{array}{c}\text { Mother's age } \\
\text { (years) }\end{array}$ & $\begin{array}{c}\text { Fetal mass } \\
(\mathbf{k g})\end{array}$ \\
\hline Group A & $22.3 \pm 1.7$ & $3.2 \pm 0.55$ \\
$(20-25$ years, $\mathrm{n}=20)$ & $(21.5-23.1)$ & $(2.9-3.5)$ \\
\hline Group B & $27.3 \pm 1.3$ & $3.2 \pm 0.48$ \\
$(26-30$ years, $\mathrm{n}=20)$ & $(26.7-27.9)$ & $(3.1-3.5)$ \\
\hline Group C & $32.6 \pm 1.4$ & $3.1 \pm 0.5$ \\
$(31-35$ years, $\mathrm{n}=20)$ & $(31.8-33.2)$ & $(2.8-3.3)$ \\
\hline Group D & $36.4 \pm 1.1$ & $2.5 \pm 0.3^{*}, \&$,\# \\
$(36-40$ years, $\mathrm{n}=20)$ & $(36.3-37.4)$ & $(2.3-2.7)$ \\
\hline Group E & $42 \pm 1.7$ & $2.9 \pm 0.69$ \\
$(41-45$ years, $\mathrm{n}=20)$ & $(41.2-42.8)$ & $(2.5-3.2)$ \\
\hline Total & $32.2 \pm 7.1$ & $3.1 \pm 0.58$ \\
$(\mathrm{n}=100)$ & $(30.8-33.6)$ & $(2.9-3.1)$ \\
\hline
\end{tabular}

Data express mean \pm SD, lower bound-upper bound of $95 \%$ confidence interval for the mean is given in parenthesis. ${ }^{*, \&, \#}$ statistically significant differences $v$ s. group A ( $<<0.001)$, group B $(\mathrm{p}<0.001)$ and group $\mathrm{C}(\mathrm{p}=0.03)$, respectively

of full-term human umbilical cord was determined using Pearson's correlation. A $p$ value less than 0.05 was considered to be statistically significant.

\section{Results}

\section{Mother's age and fetal mass}

The mean age of the mothers included in this study was $32.2 \pm 7.1$ years. There was a significant decrease in fetal weights in group D (35-40 years) compared with group A (20-25 years) (Table 1$)$. A significant negative correlation $(\mathrm{r}=0.34, \mathrm{p}=0.001)$ was found between the maternal age and the fetal weights.

\section{Immunohistochemical analysis of CD105 and CD29 expression}

Figure 1 presents microphotographs of sections of umbilical cord (UC) derived from mothers of various ages. The sections were stained by $\mathrm{H} \& \mathrm{E}$ and immunohistochemistry. It was found that UCMSCs showed CD105 and CD29 immunoexpression while they did not express CD34 surface antigen marker (data not shown). MSCs of AM and WJ showed strong cytoplasmic and nuclear expression of CD105 in groups A, B and $\mathrm{C}$ and a weak expression in the groups $\mathrm{D}$ and $\mathrm{E}$. The MSCs of AM showed moderate cytoplasmic CD29 expression in groups A and B and a weak cytoplasmic immunoreactivity in groups D and $\mathrm{E}$ (Figure 1).

Figure 2 presents immunoreactivity of CD105 and CD29 molecules in the wall of umbilical artery (UA). 


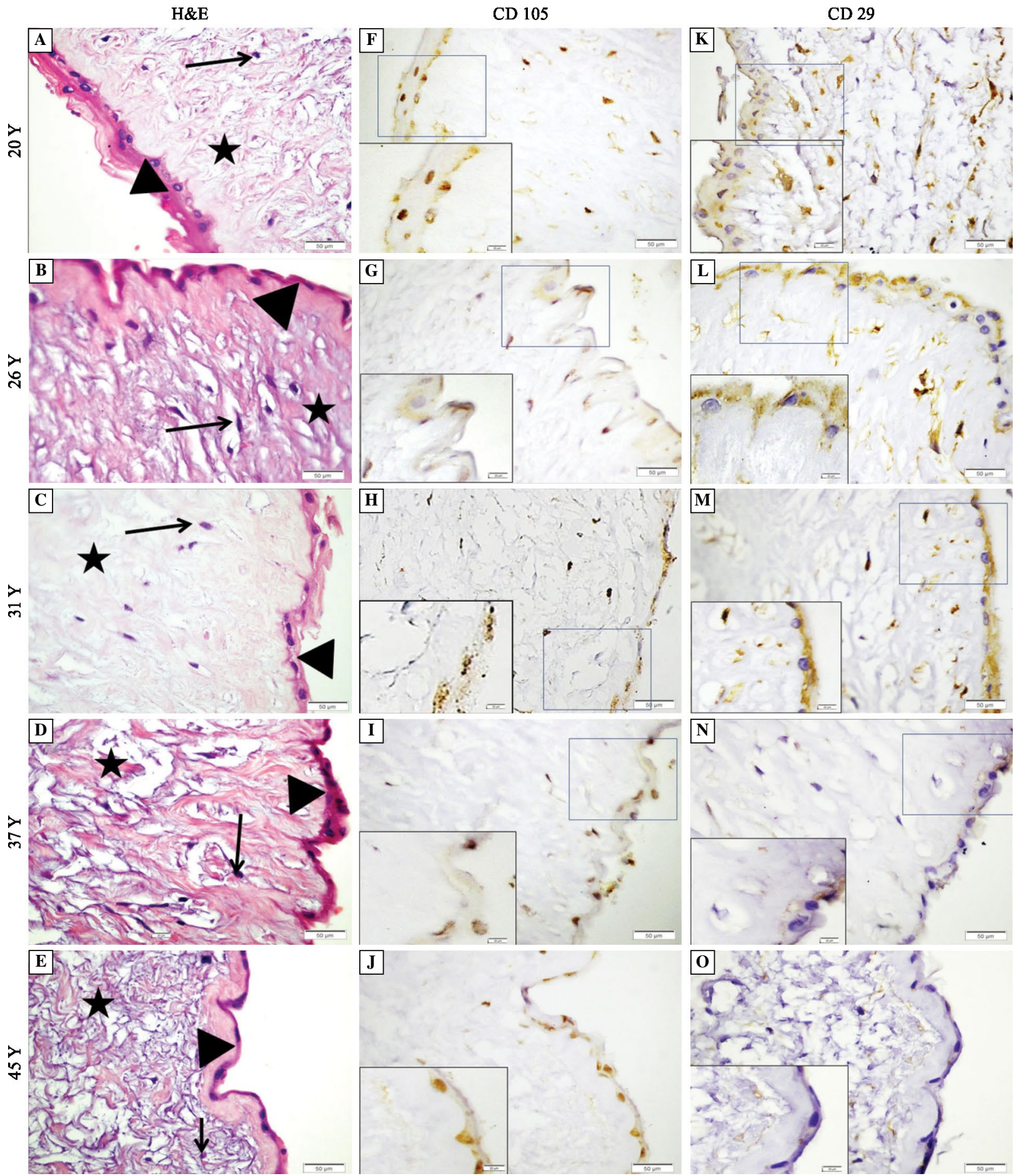

Figure 1. Immunoexpression of CD105- and CD 29-positive MSCs in amniotic membrane (AM) and Wharton's jelly (WJ) of umbilical cords from mothers of different ages. The H \& E staining (A-E) revealed that WJ is formed of gelatinous ground substance with sparse collagen bundles (star) and stellate fibroblasts (arrow) and covered by amniotic epithelium (arrowhead). MSCs in AM \& WJ show cytoplasmic and nuclear CD105 immunoexpression (brown color) which intensity decreased in older age groups (F-J). MSCs in AM \& WJ show only cytoplasmic CD29 immunoexpression (K-O) that was also reduced with age. Bars $-50 \mathrm{~mm}$, in inserts $-20 \mathrm{~mm}$, total magnification $\times 400$, inserts $\times 1000$

The endothelial cells show strong CD105 nuclear and cytoplasmic expression in groups $\mathrm{A}$ and $\mathrm{B}$, a moderate expression in groups $\mathrm{C}$ and $\mathrm{D}$, and a weak expression in group E. The endothelial cells of UA present a moderate cytoplasmic CD29 immunoexpression in group A while the other groups showed a weak 


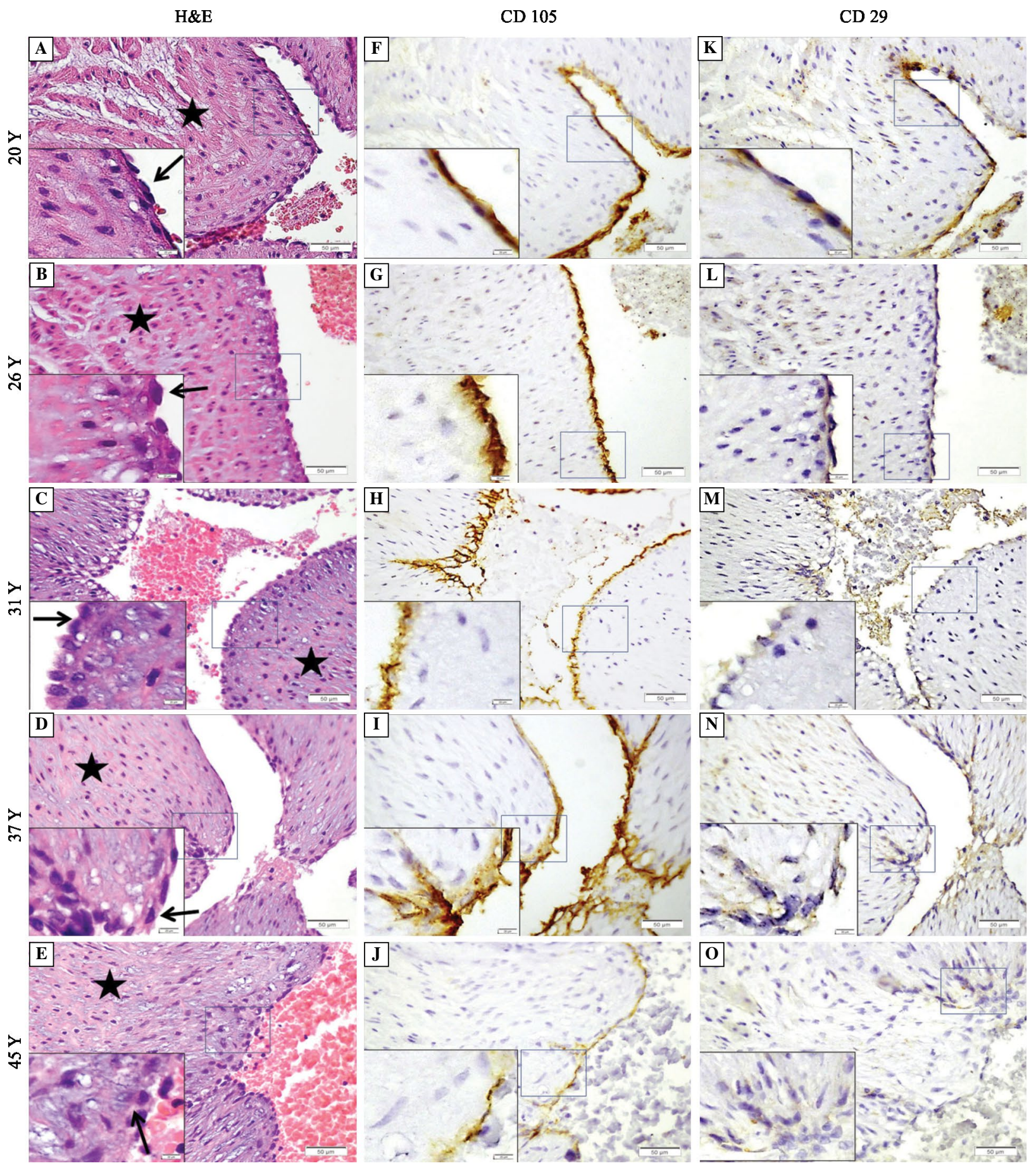

Figure 2. Immunoexpression of CD105- and CD 29-positive MSCs in umbilical cord arteries from mothers of different ages. Sections stained with H \& E (A-E) show endothelial cells (thin arrow) of tunica intima, and tunica media formed of elastic and smooth muscles (star). Tunica adventitia is not seen at this magnification. MSCs show cytoplasmic and nuclear CD105 immunoexpression (brown color) (F-J) as well as cytoplasmic CD29 immunoexpression (K-O) that appear to be reduced in the older age groups. Bars and magnifications as in the legend to Figure 1

expression of this antigen. MSCs showed cytoplasmic and nuclear CD105 immunoexpression as well as cytoplasmic CD29 immunoexpression that appear to be reduced in the older age groups.
Figure 3 shows that endothelial cells in the wall of umbilical vein (UV) exhibit strong nuclear and cytoplasmic CD105 expression in groups $\mathrm{A}, \mathrm{B}$ and $\mathrm{C}$, and a moderate expression in groups $\mathrm{D}$ and $\mathrm{E}$. The en- 

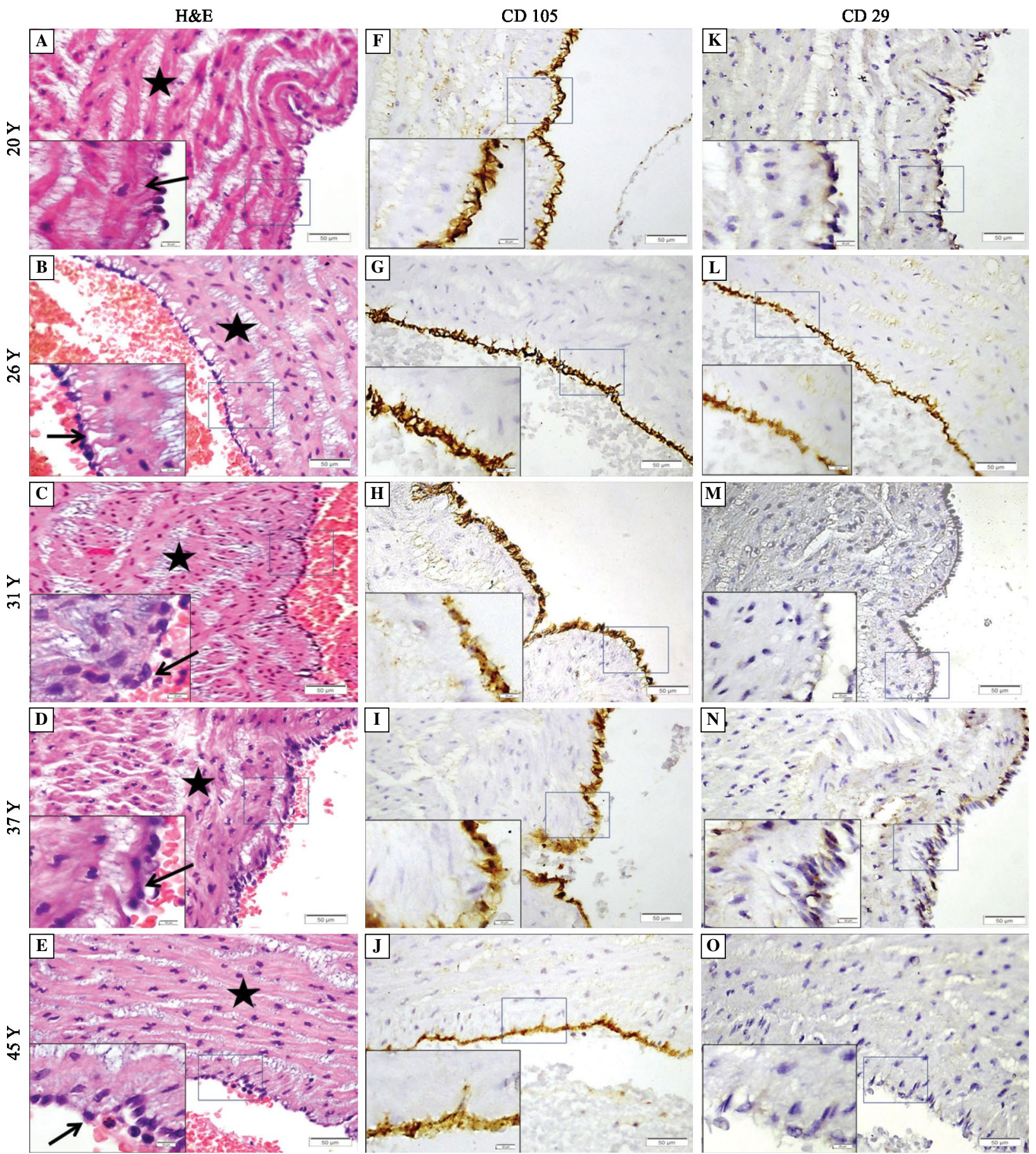

Figure 3. Immunoexpression of CD105- and CD 29-positive MSCs in umbilical cord veins from mothers of different ages. Sections stained with H \& E (A-E) show endothelial cell (thin arrow) lining and thick muscular layer (star). MSCs show stronger nuclear and cytoplasmic CD105 immunoexpression (brown color) (F-J), and cytoplasmic CD29 immunoexpression $(\mathbf{K}-\mathbf{O})$ in veins of the young age groups compared to the older ones $(\times 400$, insert $\times 1,000)$

dothelial cells also showed a moderate cytoplasmic CD29 expression in group A while in the other groups they showed a weak expression. MSCs showed stronger nuclear and cytoplasmic CD105 immunoexpression, and cytoplasmic CD29 immunoexpression in umbilical veins of the young age groups compared to the older ones.

\section{Semiquantitative assessment of CD105 and CD29 immunoreactivity}

Data presented in Table 2 show a significant decrease related to maternal age in the area percent (AP) and mean intensity (MI) of CD105 and CD29 
Table 2. Area percent (AP) and mean intensity (MI) of CD105 and CD29 immunoexpression in MSCs of the amniotic membrane and Wharton's jelly of umbilical cord

\begin{tabular}{|l|c|c|c|c|}
\hline \multirow{2}{*}{ Age groups } & \multicolumn{2}{|c|}{ AP } & \multicolumn{2}{c|}{ MI (lum) } \\
\cline { 2 - 5 } & CD105 & CD29 & CD105 & CD29 \\
\hline Group A (20-25 years, $\mathrm{n}=20)$ & $1.83 \pm 0.48$ & $4.43 \pm 0.94$ & $177.4 \pm 5.5$ & $185.2 \pm 10.4$ \\
& $(1.61-2.06)$ & $(3.99-4.88)$ & $(174.8-179.9)$ & $(180.3-189.9)$ \\
\hline Group B (26-30 years, $\mathrm{n}=$ 20) & $1.45 \pm 0.46$ & $4.25 \pm 0.99$ & $168.9 \pm 11.04$ & $173.8 \pm 19.5$ \\
& $(1.23-1.67)$ & $(3.78-4.71)$ & $(163.7-174.1)$ & $(164.2-182.9)$ \\
\hline Group C (31-35 years, $\mathrm{n}=$ 20) & $0.89 \pm 0.74$ & $3.45 \pm 0.68$ & $133.5 \pm 11.3$ & $131.7 \pm 14.6$ \\
& $(0.54-1.23)$ & $(3.13-3.77)$ & $(81.91-89.22)$ & $(124.8-138.5)$ \\
& $\mathrm{p} 1<0.001$ & $\mathrm{p} 1<0.001$ & $\mathrm{p} 1<0.001$ & $\mathrm{p} 1<0.001$ \\
& $\mathrm{p} 2=0.004$ & $\mathrm{p} 2=0.007$ & $\mathrm{p} 2<0.001$ & $\mathrm{p} 2<0.001$ \\
\hline Group D (36-40 years, $\mathrm{n}=20)$ & $0.46 \pm 0.24$ & $1.43 \pm 0.39$ & $85.57 \pm 8.03$ & $70.7 \pm 17.5$ \\
& $(0.34-0.58)$ & $(1.25-1.6)$ & $(81.9-89.2)$ & $(62.7-78.7)$ \\
& $\mathrm{p} 1<0.001$ & $\mathrm{p} 1<0.001$ & $\mathrm{p} 1<0.001$ & $\mathrm{p} 1<0.001$ \\
& $\mathrm{p} 2<0.001$ & $\mathrm{p} 2<0.001$ & $\mathrm{p} 2<0.001$ & $\mathrm{p} 2<0.001$ \\
& $\mathrm{p} 3=0.02$ & $\mathrm{p} 3<0.001$ & $\mathrm{p} 3<0.001$ & $\mathrm{p} 3=0.001$ \\
\hline Group E (41-45 years, $\mathrm{n}=20)$ & $0.30 \pm 0.12$ & $0.42 \pm 0.28$ & $68.4 \pm 13.6$ & $38.3 \pm 10.1$ \\
& $(1.2-1.7)$ & $(0.28-0.56)$ & $(61.9-74.9)$ & $(33.4-43.2)$ \\
& $\mathrm{p} 1<0.001$ & $\mathrm{p} 1<0.001$ & $\mathrm{p} 1<0.001$ & $\mathrm{p} 1<0.001$ \\
& $\mathrm{p} 2<0.001$ & $\mathrm{p} 2<0.001$ & $\mathrm{p} 2<0.001$ & $\mathrm{p} 2<0.001$ \\
& $\mathrm{p} 3=0.003$ & $\mathrm{p} 3<0.001$ & $\mathrm{p} 3<0.001$ & $\mathrm{p} 3<0.001$ \\
& $\mathrm{p} 4=0.01$ & $\mathrm{p} 4<0.001$ & $\mathrm{p} 4<0.001$ & $\mathrm{p} 4<0.001$ \\
\hline
\end{tabular}

Data are expressed as mean $\pm \mathrm{SD}$, (lower bound-upper bound of $95 \%$ confidence interval for the mean). p1, p2, p3, and p4 show significant differences vs. groups A, B, C, and D, respectively. Significance was considered at $\mathrm{p}<0.05$

immunoexpression in the AM and WJ except for the group B that did not differ significantly from group A (Table 2). Regarding the UA (Table 3), there was a significant decrease in AP and MI of CD105 and CD 29 immunoexpression in the groups C, D and E compared to group $\mathrm{A}$ as well as in the groups $\mathrm{D}$ and $\mathrm{E}$ compared to the group C. A significant decrease in these parameters was observed also in group $\mathrm{E}$ compared to the group D (Table 3).

In the umbilical vein (Table 4) there was a significant decrease in AP of CD105 and CD 29 immunoexpression in the groups $\mathrm{D}, \mathrm{E}$ compared with groups $\mathrm{A}$ as well as in the group E compared to D. The MI of CD105 and CD 29 immunoexpression in the groups C, D, E also showed significant decrease compared with groups $\mathrm{A}$ as well as in the group E compared to D (Table 4).

We found significantly negative correlations (Table 5) between the maternal age and AP and MI of both CD105 and CD29 immunoexpression in the studied components of the umbilical cord (AM and WJ, UA and UV). Moreover, it was found that CD105 positive MSCs were expressed with maximal intensity in the UA with no significant difference compared to UV whereas this expression was significantly reduced $(\mathrm{p}<0.001)$ in the AM and WJ. On the other hand, CD29 positive MSCs were maximally expressed in the WJ and AM and were significantly less intense expressed in UA and UV (Table 6).

\section{Expression of the CD105 and CD29 genes in umbilical cord components}

In the amniotic membrane and Wharton's jelly of the umbilical cord, mRNA levels of CD105 and CD29 were significantly $(\mathrm{p}<0.001)$ decreased in groups $\mathrm{C}(\sim 2$ fold/CD105 and $\sim 1.5$ fold/CD29), D $(\sim 3.5$ fold/CD105 and $\sim 2.5$ fold/CD29), and E ( $\sim 3.5$ fold/ $/ \mathrm{CD} 105$ and $\sim 3$ fold/CD29) in comparison to group A. In addition, there was a significant decrease in the expression of $C D 105$ and $C D 29$ genes in groups D $(\mathrm{p}=0.004, \mathrm{p}<0.001)$ and $\mathrm{E}(\mathrm{p}=0.005, \mathrm{p}<0.001)$ as compared to group $\mathrm{C}$ (Figure $4 \mathrm{~A})$.

Data presented in the Figure 4B show that CD105 mRNA levels were lower in the umbilical artery of the groups $\mathrm{C}(\sim 1.5$ fold $), \mathrm{D}(\sim 2$ fold $)$ and $\mathrm{E}$ ( $\sim 5$ fold $)$ as compared to group A. There was also a significant decrease $(p=0.008, p<0.001)$ of this gene expression between groups $\mathrm{D}$ and $\mathrm{E}$ compared to group $\mathrm{C}$. CD29 mRNA levels were lower in groups D $(\sim 2.5$ fold/CD29) and E ( 2.5 fold/CD29) as compared to group A (Figure 4B).

In the umbilical veins, $C D 105$ and $C D 29$ gene expression was decreased in groups $\mathrm{D}$ (both $\sim 1.5$ fold) and E ( $\sim 3.5$ fold for CD105 and $\sim 1.5$ fold for $C D 29)$ as compared to group A. The expression of both genes was also lower in UVs in groups $\mathrm{D}$ and $\mathrm{E}$ compared to group C (Figure 4C). 
Table 3. Area percent (AP) and mean intensity (MI) of CD105 and CD29 immunoexpression in MSCs of the umbilical artery of different age groups

\begin{tabular}{|l|c|c|c|c|}
\hline \multirow{2}{*}{ Age groups } & \multicolumn{2}{|c|}{ AP } & \multicolumn{2}{|c|}{ MI (lum) } \\
\cline { 2 - 5 } & CD105 & CD29 & CD105 & CD29 \\
\hline Group A (20-25 years, $\mathrm{n}=20)$ & $4.53 \pm 1.12$ & $0.49 \pm 0.18$ & $191.3 \pm 6.7$ & $118.7 \pm 9.3$ \\
& $(4-5.06)$ & $(0.36-0.63)$ & $(188.1-194.4)$ & $(114.3-123.1)$ \\
\hline Group B (26-30 years, $\mathrm{n}=20)$ & $3.87 \pm 1.23$ & $0.45 \pm 0.18$ & $186.5 \pm 6.4$ & $113.1 \pm 23.2$ \\
& $(3.30-4.45)$ & $(0.37-0.54)$ & $(183.1-189.6)$ & $(109.1-117.1)$ \\
\hline Group C (31-35 years, $\mathrm{n}=20)$ & $3.20 \pm 0.61$ & $0.37 \pm 0.16$ & $180.2 \pm 7.4$ & $58.2 \pm 10.1$ \\
& $(2.92-3.49)$ & $(0.20-0.54)$ & $(176.7-183.6)$ & $(35.4-62.9)$ \\
& $\mathrm{p} 1<0.001$ & $\mathrm{p} 1=0.03$ & $\mathrm{p} 1<0.001$ & $\mathrm{p} 1<0.001$ \\
& & & & $32<0.001$ \\
\hline Group D (36-40 years, $\mathrm{n}=20)$ & $2.17 \pm 0.37$ & $0.19 \pm 0.11$ & $178.1 \pm 8.9$ & $39.7 \pm 6.8$ \\
& $(2.91-3.49)$ & $(0.07-0.28)$ & $(174.1-182.2)$ & $(36.7-42.8)$ \\
& $\mathrm{p} 1<0.001$ & $\mathrm{p} 1=0.004$ & $\mathrm{p} 1<0.001$ & $\mathrm{p} 1<0.001$ \\
& $\mathrm{p} 2<0.001$ & $\mathrm{p} 2<0.001$ & $\mathrm{p} 2=0.003$ & $\mathrm{p} 2<0.001$ \\
& $\mathrm{p} 3<0.001$ & & & $\mathrm{p} 3<0.001$ \\
\hline \multirow{2}{*}{ Group E (41-45 years, $\mathrm{n}=20)$} & $0.94 \pm 0.30$ & $0.12 \pm 0.10$ & $156.3 \pm 5.2$ & $28.5 \pm 5.4$ \\
& $(0.79-1.08)$ & $(0.07-0.25)$ & $(153.7-158.8)$ & $(25.8-31.1)$ \\
& $\mathrm{p} 1<0.001$ & $\mathrm{p} 1<0.001$ & $\mathrm{p} 1<0.001$ & $\mathrm{p} 1<0.001$ \\
& $\mathrm{p} 2<0.001$ & $\mathrm{p} 2<0.001$ & $\mathrm{p} 2<0.001$ & $\mathrm{p} 2<0.001$ \\
& $\mathrm{p} 3<0.001$ & $\mathrm{p} 3=0.03$ & $\mathrm{p} 3<0.001$ & $\mathrm{p} 3<0.001$ \\
& $\mathrm{p} 4<0.001$ & $\mathrm{p} 4=0.04$ & $\mathrm{p} 4<0.001$ & $\mathrm{p} 4<0.001$ \\
\hline
\end{tabular}

Data are expressed as mean \pm SD (lower bound-upper bound of $95 \%$ confidence interval for the mean). p1, p2, p3, and p4 show significant differences vs. groups A, B, C, and D, respectively. Significance was considered at $\mathrm{p}<0.05$

Table 4. Area percent (AP) and mean intensity (MI) of CD105 and CD29 immunoexpression in MSCs of the umbilical vein of different age groups

\begin{tabular}{|l|c|c|c|c|}
\hline \multirow{2}{*}{ Age groups } & \multicolumn{2}{|c|}{ AP } & \multicolumn{2}{|c|}{ MI (lum) } \\
\cline { 2 - 5 } & CD105 & CD29 & CD105 & CD29 \\
\hline Group A (20-25 years, $\mathrm{n}=20)$ & $3.45 \pm 0.52$ & $0.81 \pm 0.6$ & $180.3 \pm 7.1$ & $103.6 \pm 11.9$ \\
& $(3.35-3.55)$ & $(0.78-0.84)$ & $(176.9-183.6)$ & $(98.1-109.2)$ \\
\hline Group B (26-30 years, $\mathrm{n}=20)$ & $3.63 \pm 0.53$ & $0.57 \pm 0.13$ & $174.5 \pm 6.4$ & $98.1 \pm 9.5$ \\
& $(3.19-3.52)$ & $(0.51-0.63)$ & $(171.5-177.6)$ & $(98.1-54.6)$ \\
\hline Group C (31-35 years, $\mathrm{n}=20)$ & $3.34 \pm 0.25$ & $0.51 \pm 0.31$ & $171.5 \pm 8.4$ & $50.7 \pm 4.2$ \\
& $(3.22-3.45)$ & $(0.07-0.11)$ & $(167.5-175.4)$ & $(48.8-109.2)$ \\
& & & $\mathrm{p} 1=0.003$ & $\mathrm{p} 1<0.001$ \\
\hline Group D (36-40 years, $\mathrm{n}=20)$ & $2.27 \pm 0.34$ & $0.07 \pm 0.03$ & $166.1 \pm 8.9$ & $50.7 \pm 4.2$ \\
& $(2.12-2.43)$ & $(0.06-0.08)$ & $(162.1-170.3)$ & $(48.77-52.7)$ \\
& $\mathrm{p} 1<0.001$ & $\mathrm{p} 1<0.001$ & $\mathrm{p} 1<0.001$ & $\mathrm{p} 1<0.001$ \\
& $\mathrm{p} 2<0.001$ & $\mathrm{p} 2<0.001$ & $\mathrm{p} 2=0.005$ & $\mathrm{p} 2=0.001$ \\
& $\mathrm{p} 3<0.001$ & $\mathrm{p} 3<0.001$ & & $\mathrm{p} 3<0.001$ \\
\hline Group E (41-45 years, $\mathrm{n}=20)$ & $1.03 \pm 0.019$ & $0.06 \pm 0.03$ & $144.3 \pm 5.2$ & $33.7 \pm 6.7$ \\
& $(1.05-1.05)$ & $(0.06-0.08)$ & $(141.7-146.8)$ & $(30.4-36.9)$ \\
& $\mathrm{p} 1<0.001$ & $\mathrm{p} 1<0.001$ & $\mathrm{p} 1<0.001$ & $\mathrm{p} 1<0.001$ \\
& $\mathrm{p} 2<0.001$ & $\mathrm{p} 2<0.001$ & $\mathrm{p} 2<0.001$ & $\mathrm{p} 2<0.001$ \\
& $\mathrm{p} 3<0.001$ & $\mathrm{p} 3<0.001$ & $\mathrm{p} 3<0.001$ & $\mathrm{p} 3<0.001$ \\
& $\mathrm{p} 4<0.001$ & $\mathrm{p} 4=0.001$ & $\mathrm{p} 4=0.001$ \\
\hline
\end{tabular}

Data are expressed as mean $\pm \mathrm{SD}$, (lower bound-upper bound of $95 \%$ confidence interval for the mean). p1, p2, p3, and p4 show significant differences vs. groups A, B, C, and D, respectively. Significance was considered at $\mathrm{p}<0.05$ 
Table 5. Correlations between maternal age and CD105 and CD29 immunoexpression in MSCs in different parts of umbilical cord

\begin{tabular}{|l|c|c|c|c|}
\hline \multirow{2}{*}{ Parameter } & \multicolumn{4}{|c|}{ Maternal age } \\
\cline { 2 - 5 } & \multicolumn{2}{|c|}{ AP } & \multicolumn{2}{c|}{ MI } \\
\cline { 2 - 5 } CD105 & P value & r value \\
\hline AM and WJ & -0.741 & $\mathrm{p}<0.001$ & -0.932 & $\mathrm{p}<0.001$ \\
\hline UA & -0.841 & $\mathrm{p}<0.001$ & -0.780 & $\mathrm{p}<0.001$ \\
\hline UV & -0.844 & $\mathrm{p}<0.001$ & -0.771 & $\mathrm{p}<0.001$ \\
\hline CD29 & & & $\mathrm{p}<0.001$ \\
\hline AM and WJ & -0.865 & $\mathrm{p}<0.001$ & -0.937 & $\mathrm{p}<0.001$ \\
\hline UA & -0.471 & $\mathrm{p}<0.001$ & -0.927 & $\mathrm{p}<0.001$ \\
\hline UV & -0.871 & $\mathrm{p}<0.001$ & -0.902 & \\
\hline
\end{tabular}

$\mathrm{AP}$ — area percent; MI — mean intensity; AM — amniotic membrane; WJ — Wharton's jelly; AU — umbilical artery; UV — umbilical vein. Significance was considered at $\mathrm{p}<0.05$

Table 6. Immunoexpression of CD105 and CD29 MSCs markers in different areas of human umbilical cord of all studied cases

\begin{tabular}{|l|c|c|c|c|}
\hline \multirow{4}{*}{ Area } & \multicolumn{4}{|c|}{ MSCs markers (n= 100) } \\
\cline { 2 - 5 } & \multicolumn{2}{|c|}{ CD105 } & \multicolumn{2}{c|}{ CD29 } \\
\cline { 2 - 5 } & AP & MI & AP & $120.3 \pm 58.9$ \\
\hline \multirow{2}{*}{ AM and WJ } & $0.99 \pm 0.75$ & $126.9 \pm 44.7$ & $2.81 \pm 1.74$ & $(108.5-131.9)$ \\
\hline \multirow{2}{*}{ UA } & $(0.84-1.13)$ & $(118.1-135.8)$ & $(2.4-3.1)$ & $71.8 \pm 38.3$ \\
& $2.95 \pm 1.49$ & $178.7 \pm 43.7$ & $0.33 \pm 0.28$ & $(64.2-79.4)$ \\
& $(2.66-3.25)$ & $(175.9-181.5)$ & $(0.27-0.38)$ & $\mathrm{p} 1<0.001$ \\
\hline UV & $\mathrm{p} 1<0.001$ & $\mathrm{p} 1<0.001$ & $0.31<0.001$ & $66.3 \pm 30.1$ \\
& $2.70 \pm 0.96$ & $167.6 \pm 44.2$ & $0.32 \pm 0.31$ & $(60.4-72.3)$ \\
& $(2.51-2.89)$ & $(164.7-170.4)$ & $(0.25-0.38)$ & $\mathrm{p} 2<0.001$ \\
\hline
\end{tabular}

Data are expressed as mean \pm SD (lower bound-upper bound of $95 \%$ confidence interval for the mean). p1 and p2 shows significance of UA and UV $v s$. $\mathrm{AM}$ and WJ, respectively. Significance was considered at $\mathrm{p}<0.05$

As presented in Figure 5 in the three studied components of the umbilical cord CD105 showed highest mRNA level in UA $(2.22 \pm 0.1)$ and $U V(2.13 \pm 0.1)$, and the lowest level in the AM and WJ $(0.588 \pm 0.05)$. $C D 29$ expression was the highest in UV $(1.01 \pm 0.05)$, moderate in $\mathrm{AM}$ and $\mathrm{WJ}(0.686 \pm 0.07)$ and lowest in UA $(0.252 \pm 0.05)$

\section{Discussion}

The results of this study show that CD105-, CD29-positive and CD34-negative MSCs present in the major components of human umbilical cord (AM and WJ, UA and UV) and this finding is supported by some previous studies [1, 4, 5, 25-27]. It was an interesting observation that endothelial lining of both the UA and UV showed expression of the MSCs markers CD29 and CD105. In a previous study of Fonsatti et al. endoglin (also known as CD105) was described to be highly expressed on proliferating vascular endothelial cells [28].

In the present data, a significant decrease in the AP of both CD105 and CD29 immunoexpression in the different studied area of the UC was observed in the older age groups compared with the younger ones indicated a significant reduction in the amount of cells expressing these markers. In addition, a significant decrease was observed in both CD105 and CD29 immunoexpression in the older groups compared to the younger ones which indicated a significant reduction in expression level of individual cells. A significant negative correlations existed between 

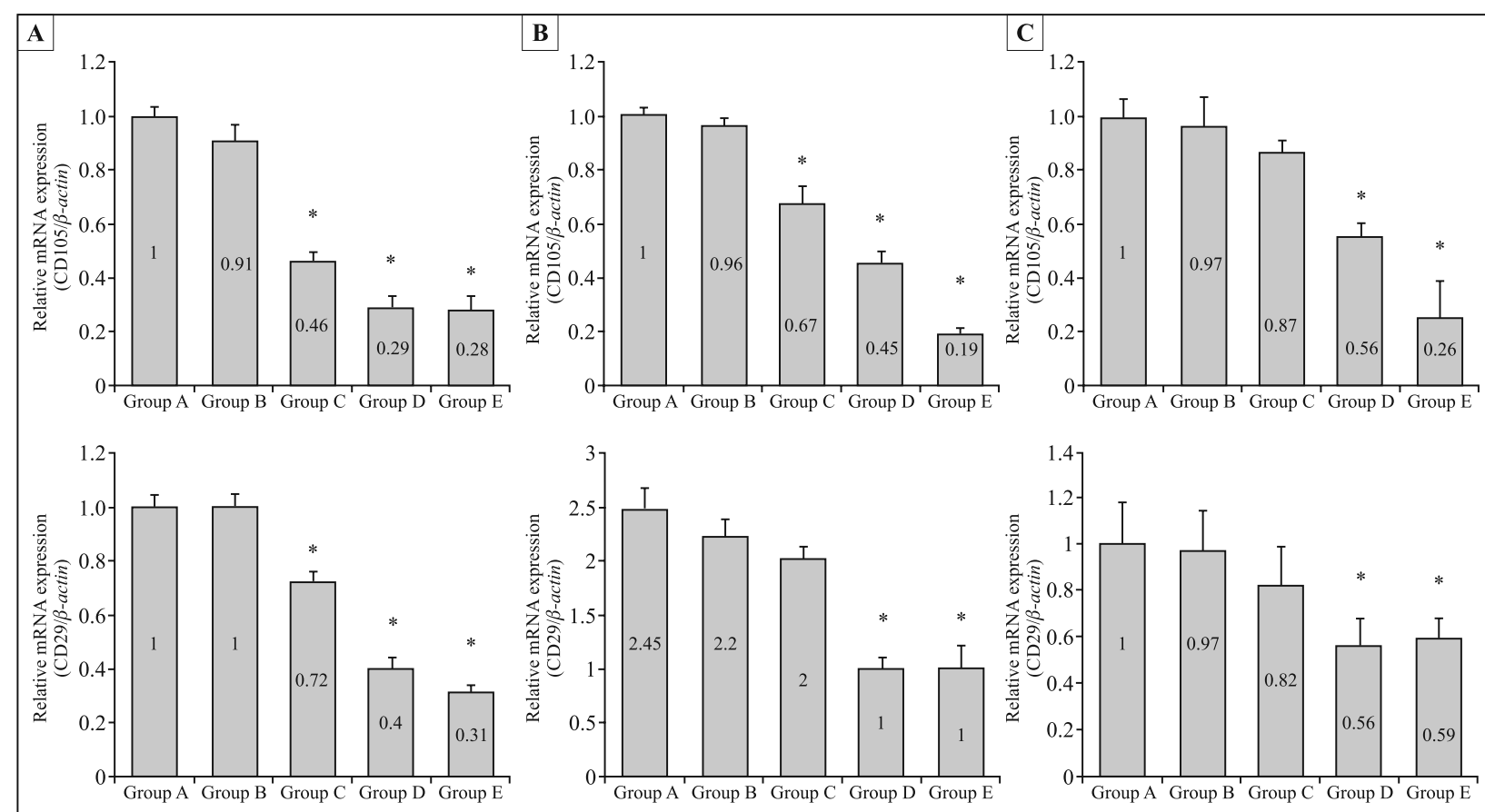

Figure 4. Real-time PCR analysis of the expression of CD105 and CD29 genes in amniotic membrane and Wharton's jelly (A), umbilical artery (B), and umbilical vein (C) isolated from human umbilical cord from mothers of various ages. The age of mothers was 20-25, 26-30, 31-35, 36-40 and 45-48 in groups A, B, C, D and E, respectively. The housekeeping gene $\beta$-actin was used as an internal reference for normalization. Data are expressed as mean fold of change \pm SEM $(\mathrm{n}=20) .{ }^{*} \mathrm{p}<0.05$ vs. group A

the maternal age and both CD105 and CD29 immunoexpression, in term of AP and MI, in different areas of the UC. Validation of immunohistochemical results was performed by quantitative RT-PCR which revealed a significant decrease in $C D 105$ and $C D 29$ genes expression in older ages compared with the younger ones. This finding could justify the reduction observed in the MSCs markers expression. Although RT-PCR was used frequently to assess the expression of MSCs CD105 [29-31] and CD29 genes [32] in human umbilical vein cells and placenta-derived stem cells, no previous studies have investigated in depth the impact of age on such MSCs gene expression in general or in umbilical cord stem cells in particular. In a previous experimental study, Asumda and Chase [33] have observed that the expression profile of Oct-4, Sox-2 and NANOG in the adult BM-MSCs derived from the bone marrow of old rats was significantly lower in comparison to that observed in the young MSC cultures. Only one study characterized human MSCs isolated from UC of different donors and did extensive testing on these cells to compare their proliferative, colony forming capacity and differentiation potential [14]. Our results partially support the data of Huang et al. [13], who reported that UCMSCs from young women may have a higher amount of cells that displayed positive markers for MSCs (CD44, CD90, and CD105) compared to the older ones although this difference was not statistically significance. The latter could be attributed to the small sample size of their study (five mothers in each group).

Huang et al. [13] implied that the umbilical cord from younger donor represents a relatively effective source of MSCs. They speculated that cells from umbilical cords of elder donor exhibit reduced differentiation capability and this might have been attributed to a declined functional status of older mothers' organs which provide a supportive role and a microenvironment that enables development of UCMSCs. The results of some ultrastructural and biochemical studies make it clear that the structure and function of the cytoplasmic organelles, particularly mitochondria, of both the oocytes and the granulosa cells, which are critical for fertilization and normal embryonic development, are markedly compromised in the oocytes of older women [34]. This finding is supported by earlier studies on the ATP content [35] and ultrastructure of human oocytes [36] as well as murine [37], bovine [38] and hamster [39] oocytes. Ju and Rudolph reported that the level of telomerase 


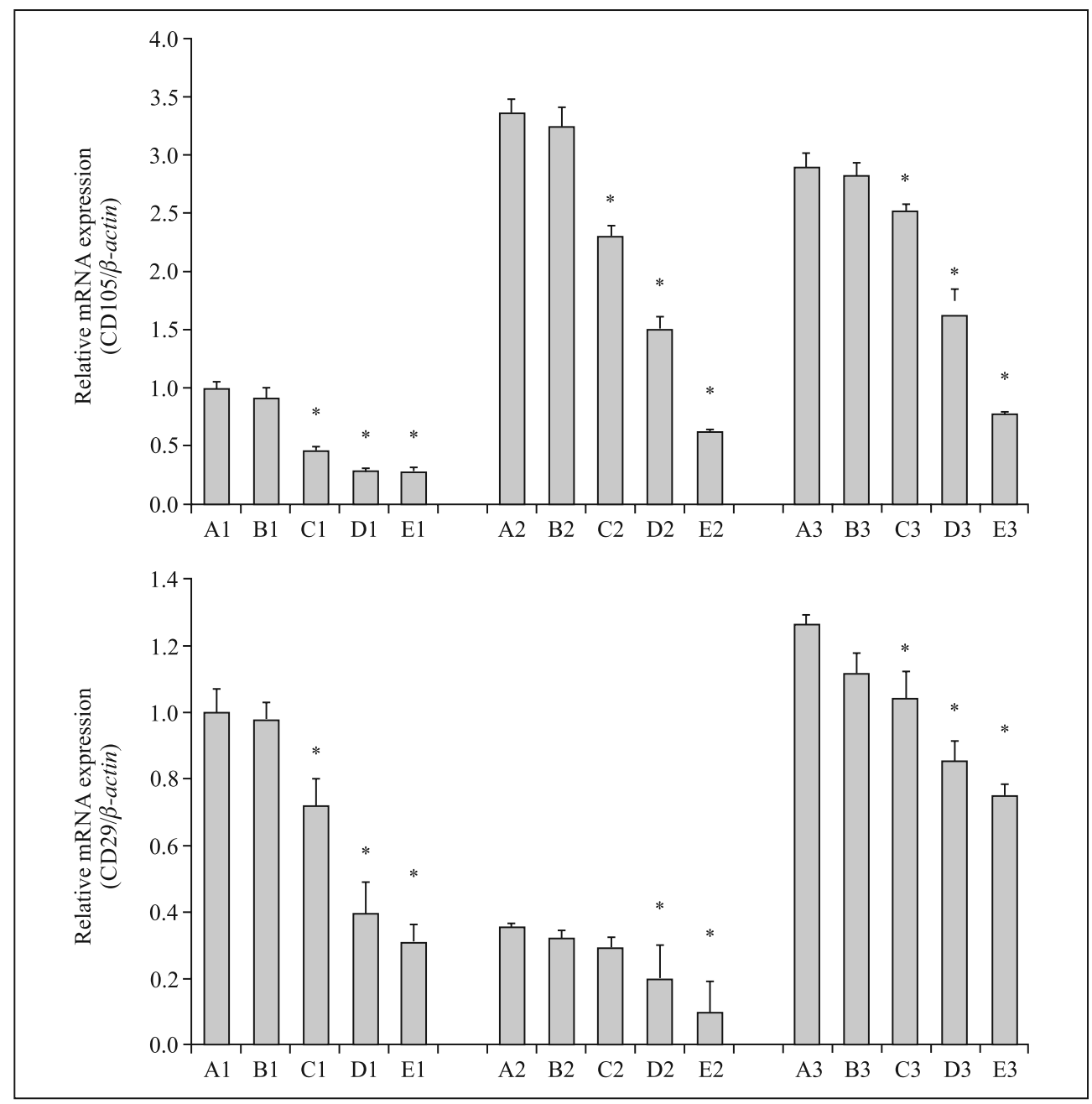

Figure 5. Comparison of the expression of CD105 and CD29 genes in amniotic layer and Wharton's jelly (A1-E1), umbilical artery (A2-E2), and umbilical vein (A3-E3) of umbilical cords from mothers of various ages assessed by real-time PCR analysis. The age groups are the same as described for Figure 4 . The housekeeping gene $\beta$-actin was used as an internal reference for normalization. Data are expressed as mean fold of change \pm SEM $(n=20) .{ }^{*} p<0.05 v s$. group A

activity is not sufficient to maintain telomere length of stem cells during aging with increased sensitivity towards senescence or apoptosis induced by telomere shortening [40]. Such findings may at least partly explain the observed decrease in the expression levels of CD105 and CD29 in UC-MSCs with increased age. The effect of age on adult stem cells was reported in few previous studies. A decrease in the number of adult MSCs in the bone marrow with increasing age had been previously reported [41]. Wagner et al. also reported an increase in size of senescent MSCs and the attenuation of the expression of specific surface markers with increasing age [42]. Yu and Kang reported that the aging of adult MSCs leads to an age-associated decline in their number, function and properties of multilineage differentiation [43]. It was demonstrated that aging caused gene expression changes in human MSCs isolated from bone marrow or mobilized peripheral blood collected from donors of different ages [44]. These changes may be triggered by many factors including intense oxidative and metabolic stress, chronic inflammation, enhanced telomere attrition and defects in DNA repair mechanisms [45].

Interestingly, the present study showed that the maximum expression of CD105 was observed in the wall of umbilical artery whereas that of CD29 was in the amniotic membrane and Wharton's jelly. This differential distribution of umbilical cord MSCs which had not been previously reported may render the selective isolation of these cells from the different areas of the UC an available option for cell therapy applications. Based on these findings, UA is recommended as a rich area of the UC with significantly large number of CD105 positive-MSCs while the AM and WJ have 
significantly large number of CD29-positive MSCs. Both areas may be easily collected from the UC.

In this study two surface antigen markers of MSCs were studied in a relatively large sample and another group of surface antigen markers could be considered in coming studies. Further studies to compare the differentiation and proliferation abilities of MSCs isolated from different areas of the UC of mothers with different ages are recommended.

In conclusion, the present study provided insights on the impact of the maternal age on CD105 and CD29 surface markers antigen and genes expressions as well as the anatomical localization of the umbilical cord MSCs. These results are of particular importance in the context of possible exchange of the UC-derived MSCs between cell banks, clinics and laboratories. Labeling the MSCs with the specific UC area from which the cells were taken as well as the mothers' age may be important to improve the outcome of therapeutic applications.

\section{References}

1. Pelosi E, Castelli G, Testa U. Human umbilical cord is a unique and safe source of various types of stem cells suitable for treatment of hematological diseases and for regenerative medicine. Blood Cells Mol Dis. 2012;49:20-28. doi: 10.1016/j. bcmd.2012.02.007.

2. Abdulrazzak H, Moschidou D, Jones $G$ et al. Biological characteristics of stem cells from foetal, cord blood and extraembryonic tissues. J R Soc Interface. 2010;7:689-706. doi: $10.1098 /$ rsif.2010.0347.

3. Mihu C, Mihu D, Costin N et al. Isolation and characterization of stem cells from the placenta and the umbilical cord. Rom J Morphol Embryol. 2008;49:441-446. PMID: 19050791.

4. Romanov A, Svintsitskaya A, Smirnov N. Searching for alternative sources of postnatal human mesenchymal stem cells: candidate MSC-like cells from umbilical cord. Stem Cells. 2003;21:105-110. doi: 10.1634/stemcells.21-1-105.

5. Wang S, Hung C, Peng T et al. Mesenchymal stem cells in the Wharton's jelly of the human umbilical cord. Stem Cells. 2004;22:1330-1337. doi: 10.1634/stemcells.2004-0013.

6. Weiss L, Medicetty S, Bledsoe R et al. Human umbilical cord matrix stem cells: preliminary characterization and effect of transplantation in a rodent model of Parkinson's disease. Stem Cells. 2006;24:781-792. doi: 10.1634/stemcells.2005-0330.

7. Sarugaser R, Lickorish D, Baksh D et al. Human umbilical cord perivascular (HUCPV) cells: a source of mesenchymal progenitors. Stem Cells. 2005;23:220-229. doi: 10.1634/stemcells.2004-0166.

8. Horwitz M, Le-Blanc K, Dominici M et al. Clarification of the nomenclature for MSC: The International Society for Cellular Therapy position statement. Cytotherapy. 2005;7:393-395. doi: 10.1080/14653240500319234

9. Dominici M, Blanc K, Mueller I et al. Minimal criteria for defining multipotentmesenchymal stromal cells. The International Society for Cellular Therapy position statement. $C y$ totherapy. 2006;8:315-317. doi: 10.1080/14653240600855905.

10. Ishige I, Nagamura T, Honda $\mathbf{J}$ et al. Comparison of mesenchymal stem cells derived from arterial, venous, and Wharton's jelly explants of human umbilical cord. Int J Hematol. 2009;90:261-269. doi: 10.1007/s12185-009-0377-3.
11. Anzalone R, Iacono L, Corrao S et al. New emerging potentials for human Wharton's jelly mesenchymal stem cells: immunological features and hepatocyte-like differentiative capacity. Stem Cells Dev. 2010;19:423-438. doi: 10.1089/scd.2009.0299.

12. Hou T, Xu J, Wu X et al. Umbilical cord Wharton's jelly: a new potential cell source of mesenchymal stromal cells for bone tissue engineering. Tissue Eng. 2009;15:2325-2334. doi: 10.1089/ten.tea.2008.0402.

13. Huang $\mathrm{S}$, Feng $\mathrm{C}, \mathrm{Wu}$ Yet al. Dissimilar characteristics of umbilical cord mesenchymal stem cells from donors of different ages. Cell Tiss Bank. 2013;14:707-713. doi: $10.1007 /$ s10561-013-9364-2.

14. Gonzalez R, Griparic L, Umana M et al. An efficient approach to isolation and characterization of Pre- and postnatal umbilical cord lining stem cells for clinical applications. Cell Transp. 2010;19:1439-1449. doi: 10.3727/096368910X514260.

15. Morrison SJ, Spradling A. Stem cells and niches: mechanisms that promote stem cell maintenance throughout life. Cell. 2008;132:598-611. doi: 10.1016/i.cell.2008.01.038.

16. Pinto M, Moraes CT. Mechanisms linking mtDNA damage and aging. Free Radic Biol Med. 2015;85:250-258. doi: 10.1016/j.freeradbiomed.2015.05.005.

17. Wong TY, Solis MA, Chen YH, Huang LL. Molecular mechanism of extrinsic factors affecting anti-aging of stem cells. World J Stem Cells. 2015;7:512-520. doi: 10.4252/wjsc. v7.i2.512.

18. Sperka T, Wang J, Rudolph KL. DNA damage checkpoints in stem cells, ageing and cancer. Nat Rev Mol Cell Biol. 2012;13:579-590. doi: 10.1038/nrm3420.

19. Hadlock FP, Shah YP, Kanon DJ et al. Fetal crown-rump length: reevaluation of relation to menstrual age (5-18 weeks) with high resolution real-time US. Radiol. 1992;182:501-505. doi: 10.1148/radiology.182.2.1732970.

20. Bancroft JD and Gamble M. Theory and practice of histological techniques. $6^{\text {th }}$ ed. Philadelphia, PA: Churchill Livingstone; 2008.

21. Thomasse BMS. Handbook of immunochemical staining methods. $3^{\text {rd }}$ ed. California: Dako Corporation Carpinteria; 2001.

22. Miller RT. Technical immunohistochemistry: achieving reliability and reproducibility of immunostains. Presented at the Society for Applied Immunohistochemsitry. New York: Flushing; 2001.

23. Castrechini N, Murhi P, Gud N et al. Mesenchymal stem cells in human placental chorionic villi reside in a vascular niche. Placenta. 2010;31:203-212. doi: 10.1016/j.placenta.2009.12.006.

24. Decaestecker C, Lopez XM, D'Haene N et al. Requirements for the valid quantification of immunostains on tissue microarray materials using image analysis. Proteomics. 2009;9:4478-4494. doi: 10.1002/pmic.200800936.

25. Montanucci P, Basta G, Pescara T et al. New simple and rapid method for purification of mesenchymal stem cells from the human umbilical cord Wharton's Jelly. Tissue Eng. 2001;17:2651-2661. doi: 10.1089/ten.TEA.2010.0587.

26. Taghizadeh R, Cetrulo K, Cetrulo C. Wharton's Jelly stem cells: future clinical applications. Placenta. 2011;32:311-315. doi: $\underline{10.1016 / j . p l a c e n t a .2011 .06 .010 .}$.

27. Alviano F, Fossati V, Marchionni C et al. Term amniotic membrane is a high throughput source for multipotentmesenchymal stem cells with the ability to differentiate into endothelial cells in vitro. BMC Develop Biol. 2007;7:7-11. doi: 10.1186/1471-213X-7-11.

28. Fonsatti E, Jekunen $\mathrm{P}$, Kairemo J et al. Endoglin is a suitable target for efficient imaging of solid tumors: in vivo evidence in 
a canine mammary carcinoma model. Clin Cancer Res. 2000; 6:2037-2043. PMID: 10815930.

29. Munaut C, Lorquet $\mathrm{S}$, Christel et al. Hypoxia is responsible for soluble vascular endothelial growth factor receptor-1 (VEGFR-1) but not for soluble endoglin induction in villous trophoblast. Human Reprod. 2008;23:1407-1415. doi: 10.1093/ humrep/den114.

30. Sitras V, Paulssen RH, Grønaas $\mathrm{H}$ et al. Differential placental gene expression in severe preeclampsia. Placenta. 2009;30:424-433. doi: 10.1016/j.placenta.2009.01.012.

31. Lappas M. Markers of endothelial cell dysfunction are increased in human omental adipose tissue from women with pre-existing maternal obesity and gestational diabetes. Metabolism. 2014;63:860-873. doi: 10.1016/j.metabol.2014.03.007.

32. Meinhardt G, Haider S, Haslinger P et al. Wnt-dependent T-cell factor-4 controls human extravillous trophoblast motility. Endocrinol. 2014;155:1908-1920. doi: 10.1210/en.2013-2042.

33. Asumda FZ, Chase PB. Age-related changes in rat bone -marrow mesenchymal stem cell plasticity. BMC Cell Biol. 2011;12:44. doi: 10.1186/1471-2121-12-44.

34. Ford JH. Reduced quality and accelerated follicle loss with female reproductive aging - does decline in theca dehydroepiandrosterone (DHEA) underlie the problem? J Biomed Sci. 2013;20:93. doi: 10.1186/1423-0127-20-93.

35. Zhao J, Li Y. Adenosine triphosphate content in human unfertilized oocytes, undivided zygotes and embryos unsuitable for transfer or cryopreservation. J Int Med Res. 2012;40:734-739. PMID: 22613437.

36. De-Bruin JP, Dorland M, Spek ER et al. Age-related changes in the ultrastructure of the resting follicle pool in human ovaries. Biol Reprod. 2004;70:419-424. doi: 10.1095/ biolreprod.103.015784
37. Kushnir VA, Ludaway T, Russ R et al. Reproductive aging is associated with decreased mitochondrial abundance and altered structure in murine oocytes. J Assist Reprod Genet. 2012;29:637-642. doi: 10.1007/s10815-012-9771-5.

38. Sugimura S, Matoba S, Hashiyada Y et al. Oxidative phosphorylation-linked respiration in individual bovine oocytes. J Reprod Dev. 2012;58:636-641. doi: 10.1262/jrd.2012-082.

39. Simsek-Duran F, Li F, Ford W. Age associated metabolic and morphologic changes in mitochondria of individual mouse and hamster oocytes. PLoS One. 2013;8:1-7. doi: 10.1371/ journal.pone.0064955.

40. Mimeault M, Batra SK. Great promise of tissue-resident adult stem/progenitor cells in transplantation and cancer therapies. Adv Exp Med Biol. 2012;741:171-186. doi: 10.1007/978-14614-2098-9 12.

41. Levi BP, Morrison SJ. Stem cells use distinct self-renewal programs at different ages. Cold Spring Harb Symp Quant Biol. 2008;73:539-553. doi: 10.1101/sqb.2008.73.049.

42. Wagner W, Bork S, Horn P et al. Aging and replicative senescence have related effects on human stem and progenitor cells. PLoS One. 2009;4:1-13. doi: 10.1371/journal.pone.0005 $\underline{846}$.

43. Yu K, Kang K. Aging-related genes in mesenchymal stem cells: a mini-review. Gerontol. 2013;59:557-563. doi: 10.1159/000353857.

44. Wagner W, Wein F, Seckinger A et al. Comparative characteristics of mesenchymal stem cells from human bone marrow, adipose tissue, and umbilical cord blood. Exp Hematol. 2005;33:1402-1416. PMID: 16263424.

45. Wagner W, Horn P, Castoldi M et al. Replicative senescence of mesenchymal stem cells - a continuous and organized process. PLoS One. 2008;5:1-12. doi: 10.1371/journal.pone.0002213.

Submitted: 6 April, 2015

Accepted after reviews: 1 September, 2015

Available as AoP: 4 September, 2015 